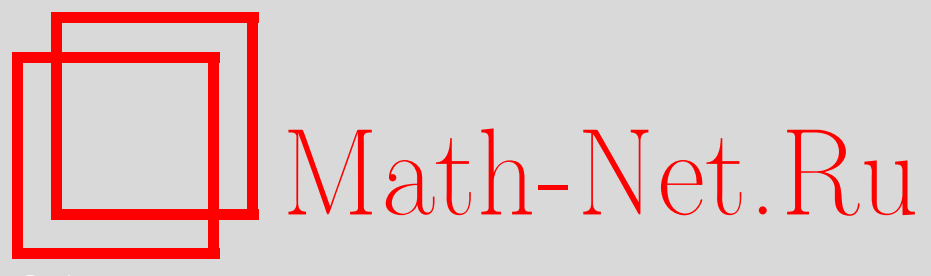

А. О. Ремизов, Геодезические на двумерных поверхностях с псевдоримановой метрикой: особенности смены сигнатуры, Матем. сб., 2009, том 200, номер 3, 75-94

DOI: https://doi.org/10.4213/sm5151

Использование Общероссийского математического портала Math-Net.Ru подразумевает, что вы прочитали и согласны с пользовательским соглашением http://www . mathnet.ru/rus/agreement

Параметры загрузки:

IP : 107.22 .136 .117

26 апреля 2023 г., 11:14:50

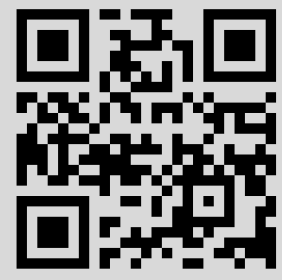




\section{А. О. Ремизов \\ Геодезические на двумерных поверхностях с псевдоримановой метрикой: особенности смены сигнатуры}

Рассматриваются гладкие двумерные поверхности с псевдоримановой метрикой, т.е. с квадратичной формой на касательном расслоении, не являющейся положительно определенной. Точки, в которых форма вырождается, называются параболическими. Рассматриваются геодезические, индуцированные данной псевдоримановой метрикой в окрестности типичных параболических точек, получены их фазовые портреты и исследованы экстремальные свойства.

Библиография: 23 названия.

Ключевые слова: псевдориманова метрика, геодезические, особые точки, резонансы, нормальные формы.

\section{§ 1. Введение}

В квантовой теории гравитации и космологии рассматриваются пространства с индефинитной метрикой, являющейся евклидовой в одной части пространства и псевдоевклидовой в другой его части. Особенно важную роль при этом играют переходы из одной части пространства в другую с изменением сигнатуры метрики - так называемые $P$ - $U$-переходы [1], или евклидово-лоренцевы переходы [2]. ${ }^{1}$

На $k$-мерном многообразии $S$, вложенном в такое пространство, или в псевдоевклидово пространство с постоянной сигнатурой (например, в пространство Минковского), метрика объемлющего пространства индуцирует псевдориманову метрику, которая меняет сигнатуру и вырождается на некотором $(k-1)$ мерном подмногообразии. Такие псевдоримановы метрики и различные их физические аспекты рассматривались многими авторами (см., например, [3]-[9]).

В настоящей статье рассматривается одна чисто математическая задача, мотивированная этой проблемой: исследуются геодезические на двумерных поверхностях с псевдоримановой метрикой $Q$ в окрестности точек, где $Q$ вырождается общим образом. ${ }^{2}$

Работа выполнена при поддержке гранта SFRH/BPD/26138/2005 (Португалия).

${ }^{1}$ А.Д. Сахаров [1] обозначает через $\sigma$ число временнь́х координат пространственно-временного континуума. Область с сигнатурой $\sigma=1$ (которая, по-видимому, включает в себя наблюдаемую Вселенную) он обозначает через $U$ (от слова Universe), а область с сигнатурой $\sigma=0$ (так называемый "мир без времени и движения") - через $P$ (от имени древнегреческого философа Парменида).

${ }^{2}$ После сдачи статьи в редакцию В. И. Арнольд сообщил автору, что аналогичная задача возникала в работах Бляшке и Эли Картана. Кроме того, рецензент статьи обратил внимание на то, что в [10] и [11] рассматриваются похожие вырождения и особые точки векторных полей. 


\section{§ 2. Уравнение геодезических и его особые точки}

Рассмотрим поверхность $S$ с гладкими криволинейными координатами $(t, x)$ и псевдоримановой метрикой - индефинитной квадратичной формой

$$
Q(d t, d x)=a(t, x) d x^{2}+2 b(t, x) d x d t+c(t, x) d t^{2},
$$

коэффициенты $a, b, c$ которой - гладкие (класса $C^{\infty}$ ) функции на $S$. Точнее говоря, в некоторых точках поверхности $S$ квадратичная форма $Q$ может быть положительно (или отрицательно) определенной, в некоторых - знакопеременной, а в некоторых - вырожденной. Точки первого типа называются эллиптическими, точки второго типа - гиперболическими. В точках вырождения дискриминант $\Delta=b^{2}-a c$ формы $Q$ обращается в нуль, при этом ее ранг может быть равен 1 или 0 . Последний случай мы исключим из рассмотрения: если коэффиценты $a, b, c$ в (1) задаются независимо, он соответствует вырождению коразмерности 3 , а в некоторых важных случаях (например, если $S-$ гладкая двумерная поверхность в псевдоевклидовом пространстве размерности $n \geqslant 3$ с метрикой $\left.d s^{2}=d x_{1}^{2}+\cdots+d x_{n-1}^{2}-d x_{n}^{2}\right)$ вообще не встречается. Таким образом, в точке вырождения выполнено равенство $b^{2}-a c=0$ и по крайней мере один из коэффициентов $a, b, c$ отличен от нуля. Без ограничения общности далее можем считать, что $a \neq 0$, так как неравенство $b \neq 0$ влечет $a c \neq 0$, а случай $c \neq 0$ превращается в $a \neq 0$ перестановкой координат $t$ и $x$. Такие точки называются параболическими.

Предположим, что в рассматриваемой области уравнение $\Delta=0$ определяет на плоскости $(t, x)$ гладкую кривую $\mathscr{D}$, которая называется линией вырождения (линией смены типа или дискриминантной кривой) и разбивает поверхность $S$ на эллиптическую $(\Delta<0)$ и гиперболическую $(\Delta>0)$ области. В эллиптической области форма $Q$ задает обычную риманову метрику (при $a>0$ ) или риманову метрику, умноженную на -1 (при $a<0$ ); в гиперболической области - псевдориманову с постоянной сигнатурой; на кривой $\mathscr{D}$ квадратичная форма $Q$ вырождается.

Рассмотрим геодезические, порожденные метрикой (1). Такими геодезическими являются экстремали уравнения Эйлера-Лагранжа с лагранжианом

$$
L=\sqrt{F}, \quad \text { где } F(t, x, p)=a(t, x) p^{2}+2 b(t, x) p+c(t, x), p=\frac{d x}{d t} .
$$

Общее уравнение Эйлера-Лагранжа имеет вид

$$
\frac{d x}{d t}=p, \quad \frac{d p}{d t}=\frac{L_{x}^{\prime}-L_{t p}^{\prime \prime}-p L_{x p}^{\prime \prime}}{L_{p p}^{\prime \prime}}
$$

и порождает в пространстве $(t, x, p)$ поле

$$
\dot{t}=L_{p p}^{\prime \prime}, \quad \dot{x}=p L_{p p}^{\prime \prime}, \quad \dot{p}=L_{x}^{\prime}-L_{t p}^{\prime \prime}-p L_{x p}^{\prime \prime},
$$

где точка сверху означает дифференцирование по некоторой независимой переменной, играющей роль времени. Записи (3) и (4) отвечают соответственно использованию неоднородных и однородных координат в пространстве, касательном к $(t, x, p)$. 
Заметим, что уравнения (3) и (4), а также вытекающие из них формулы (5)-(7) формально имеют смысл лишь при $F \neq 0$, т.е. отвечают неизотропным геодезическим, для которых равенство $F=0$ либо вообще не имеет места, либо выполнено в отдельных изолированных точках, к которым, следовательно, возможен предельный переход с условием $F \neq 0$. Это обстоятельство показывает, что возможность существования изотропных геодезических должна быть исследована отдельно (см. теорему 1).

Подставляя вытекающие из (2) выражения

$$
\begin{gathered}
L_{x}^{\prime}=\frac{F_{x}^{\prime}}{2 F^{1 / 2}}, \quad L_{t p}^{\prime \prime}=\frac{2 F F_{t p}^{\prime \prime}-F_{t}^{\prime} F_{p}^{\prime}}{4 F^{3 / 2}}, \\
L_{x p}^{\prime \prime}=\frac{2 F F_{x p}^{\prime \prime}-F_{x}^{\prime} F_{p}^{\prime}}{4 F^{3 / 2}}, \quad L_{p p}^{\prime \prime}=\frac{2 F F_{p p}^{\prime \prime}-F_{p}^{2}}{4 F^{3 / 2}}=-\frac{\Delta}{F^{3 / 2}}
\end{gathered}
$$

в (3) и (4), получаем уравнения

$$
\frac{d x}{d t}=p, \quad \frac{d p}{d t}=\frac{2 F\left(F_{x}^{\prime}-F_{t p}^{\prime \prime}-p F_{x p}^{\prime \prime}\right)+F_{p}^{\prime}\left(F_{t}^{\prime}+p F_{x}^{\prime}\right)}{2 F F_{p p}^{\prime \prime}-F_{p}^{2}}=\frac{M}{2 \Delta}
$$

и соответствующее поле

$$
\dot{t}=-\Delta F^{-3 / 2}, \quad \dot{x}=-p \Delta F^{-3 / 2}, \quad \dot{p}=-\frac{1}{2} M F^{-3 / 2},
$$

где

$$
M=-\frac{1}{2}\left[2 F\left(F_{x}^{\prime}-F_{t p}^{\prime \prime}-p F_{x p}^{\prime \prime}\right)+F_{p}^{\prime}\left(F_{t}^{\prime}+p F_{x}^{\prime}\right)\right]=\sum_{i=0}^{3} \mu_{i}(t, x) p^{i}
$$

- кубический многочлен от $p$ с коэффициентами

$$
\begin{gathered}
\mu_{3}=a\left(a_{t}^{\prime}-2 b_{x}^{\prime}\right)+b a_{x}^{\prime}, \quad \mu_{2}=b\left(3 a_{t}^{\prime}-2 b_{x}^{\prime}\right)+c a_{x}^{\prime}-2 a c_{x}^{\prime}, \\
\mu_{1}=b\left(2 b_{t}^{\prime}-3 c_{x}^{\prime}\right)+2 c a_{t}^{\prime}-a c_{t}^{\prime}, \quad \mu_{0}=c\left(2 b_{t}^{\prime}-c_{x}^{\prime}\right)-b c_{t}^{\prime} .
\end{gathered}
$$

Параболические точки являются особыми точками для уравнения геодезических: в них знаменатель в правой части второго уравнения в (6) обращается в нуль, поле (7) вертикально ${ }^{3}$ и касается ядра проектирования на плоскость $(t, x)$ или вообще не определено.

Наконец, параболические точки являются особыми и для уравнения

$$
F(t, x, p)=a(t, x) p^{2}+2 b(t, x) p+c(t, x)=0, \quad p=\frac{d x}{d t},
$$

которое определяет изотропные линии, т.е. кривые, которые в каждой точке касаются светового конуса. Длина изотропных линий в метрике (1) тождественно равна нулю. Для фиксированной точки $q$ поверхности $S$ уравнение $F(t, x, p)=0$ относительно $p$ является уравнением светового конуса, приложенного к данной точке. Если точка $q$ эллиптическая, то световой конус пуст, и все векторы касательного пространства $T_{q} S$ либо времениподобны $(a>0)$, либо пространственноподобны $(a<0)$. Геодезические, выходящие из точки $q$ со

\footnotetext{
${ }^{3}$ Вертикалъным направлением в пространстве $(t, x, p)$ будем называть направление оси $p$.
} 
всевозможными касательными направлениями, имеют (по крайней мере, локально) либо вещественную, либо чисто мнимую длину. Если точка $q$ гиперболическая, то световой конус состоит из двух различных прямых, касающихся двух выходящих из $q$ изотропных линий, и содержит внутри себя времениподобные векторы $(F>0)$, а вне себя - пространственноподобные $(F<0)$. Наконец, в параболической точке $q$ уравнение $F(t, x, p)=0$ имеет одно решение $p_{0}=-\frac{b}{a}$, световой конус вырождается и состоит из одной прямой на плоскости $T_{q} S$, при этом все остальные касательные векторы являются либо времениподобными $(a>0)$, либо пространственноподобными $(a<0)$.

Уравнение (10) относится к хорошо известному типу уравнений, не разрешенных относительно производной, называемых бинарными; 1-графики его интегральных кривых лежат на поверхности $\mathscr{F}$, задаваемой уравнением $F(t, x, p)=0$ в пространстве $(t, x, p)$, и являются траекториями системы

$$
\frac{d x}{d t}=p, \quad \frac{d p}{d t}=-\frac{F_{t}^{\prime}+p F_{x}^{\prime}}{F_{p}^{\prime}},
$$

которой соответствует поле

$$
\dot{t}=F_{p}^{\prime}, \quad \dot{x}=p F_{p}^{\prime}, \quad \dot{p}=-\left(F_{t}^{\prime}+p F_{x}^{\prime}\right) .
$$

ТЕОРема 1. Изотропные линии являются геодезическими тождественно нулевой длины, т.е. экстремалями уравнения Эйлера-Лагранжа с лагранжианом (2), в каждой точке касающимися светового конуса.

ДокАЗАТЕЛЬСтво. По определению изотропной линии ее 1-график лежит на поверхности $\mathscr{F}$ и является решением системы (11). Нужно показать, что тогда он также является и решением системы (6), для чего достаточно установить, что вторые уравнения этих систем в точках поверхности $\mathscr{F}$ совпадают. Действительно, переписывая соотношение

$$
\frac{2 F\left(F_{x}^{\prime}-F_{t p}^{\prime \prime}-p F_{x p}^{\prime \prime}\right)+F_{p}^{\prime}\left(F_{t}^{\prime}+p F_{x}^{\prime}\right)}{2 F F_{p p}^{\prime \prime}-F_{p}^{2}}=-\frac{F_{t}^{\prime}+p F_{x}^{\prime}}{F_{p}^{\prime}}
$$

в виде равенства произведений крайних и средних членов пропорции и приводя подобные слагаемые, получаем эквивалентное соотношение

$$
2 F\left[F_{p}^{\prime}\left(F_{x}^{\prime}-F_{t p}^{\prime \prime}-p F_{x p}^{\prime \prime}\right)+F_{p p}^{\prime \prime}\left(F_{t}^{\prime}+p F_{x}^{\prime}\right)\right]=0,
$$

которое выполнено во всех точках поверхности $\mathscr{F}$, заданной уравнением $F=0$.

Другими словами, теорема 1 утверждает, что $\mathscr{F}$ является интегральной поверхностью системы (6), и интегральные кривые поля направлений, высекаемого на $\mathscr{F}$ полем контактных плоскостей $d x-p d t=0$, являются траекториями обеих систем (6) и (11). Теорема 1 вместе со стандартной теоремой существования и единственности позволяет получить важное следствие: при прохождении через любую не параболическую точку геодезические сохраняют свой тип, т.е. каждая геодезическая является времениподобной, пространственноподобной или изотропной на любом интервале, не содержащем параболических точек (смена типа геодезической может произойти только в них).

Для того чтобы представить себе поведение геодезических в окрестности параболической точки, нужно наложить некоторые дополнительные условия. Мы будем предполагать, что в рассматриваемой параболической точке: 
1) коэффициент $a(t, x)$ не равен нулю;

2) дискриминант $\Delta(t, x)$ имеет ненулевой градиент;

3) изотропное направление $p_{0}=-\frac{b}{a}$ трансверсально дискриминантной кривой $\mathscr{D}$, т.е. $a \Delta_{t}^{\prime}-b \Delta_{x}^{\prime} \neq 0$.

Параболическую точку $q$, удовлетворяющую условиям 1)-3), мы будем называть типичной. В соответствующей ей точке $\left(q, p_{0}\right)$ поверхность $\mathscr{F}$ гладкая, ее проектирование $\pi$ на плоскость $(t, x)$ вдоль оси $p$ является складкой и поле $(12)$ вертикально $\left(F_{p}^{\prime}=0\right.$ и $\left.F_{t}^{\prime}+p F_{x}^{\prime} \neq 0\right)$. Действительно, так как в точке $q$ обращается в нуль дискриминант $\Delta$ квадратного трехчлена $F$ относительно $p$, то его единственный корень $p_{0}=-\frac{b}{a}$ обращает в нуль и его производную $F_{p}^{\prime}$. Вычисление $F_{t}^{\prime}+p F_{x}^{\prime}$ в точке $\left(q, p_{0}\right)$ с учетом вытекающего из $\Delta=0$ соотношения $b^{2}=a c$ дает

$$
\begin{aligned}
F_{t}^{\prime}+p F_{x}^{\prime} & =a_{t}^{\prime} p_{0}^{2}+2 b_{t}^{\prime} p_{0}+c_{t}^{\prime}+p_{0}\left(a_{x}^{\prime} p_{0}^{2}+2 b_{x}^{\prime} p_{0}+c_{x}^{\prime}\right) \\
& =-a^{-2}\left[a^{-1} b^{3} a_{x}^{\prime}-b^{2}\left(a_{t}^{\prime}+2 b_{x}^{\prime}\right)+a b\left(2 b_{t}^{\prime}+c_{x}^{\prime}\right)-a^{2} c_{t}^{\prime}\right] \\
& =-a^{-2}\left[b c a_{x}^{\prime}-a c a_{t}^{\prime}-2 b^{2} b_{x}^{\prime}+2 a b b_{t}^{\prime}+a b c_{x}^{\prime}-a^{2} c_{t}^{\prime}\right] \\
& =-a^{-2}\left[a\left(2 b b_{t}^{\prime}-a_{t}^{\prime} c-a c^{\prime} t\right)-b\left(2 b b^{\prime} x-a_{x}^{\prime} c-a c_{x}^{\prime}\right)\right] \\
& =-a^{-2}\left[a \Delta_{t}^{\prime}-b \Delta_{x}^{\prime}\right] \neq 0 .
\end{aligned}
$$

Таким образом, в окрестности точки $\left(q, p_{0}\right)$ уравнение $(10)$ имеет нормальную форму Чибрарио $p^{2}+t=0$ (см. [12]-[14]), и все его интегральные кривые представляют собой полукубические параболы с точками возврата на дискриминантной кривой $\mathscr{D}$. В случае общего положения кривая $\mathscr{D}$ состоит из типичных параболических точек, за исключением изолированных точек, в которых нарушается условие 3 ), т.е. изотропное направление $p_{0}$ касается кривой $\mathscr{D}$. $\mathrm{B}$ таких точках поле (12) общего положения имеет на поверхности $\mathscr{F}$ особую точку одного из трех типов: узел, седло или фокус. При проектировании $\pi$ соответствующая точка $q$ на плоскости $(t, x)$ называется сложенным узлом, сложенным седлом, сложенным фокусом (см. [14], [15]). Такие параболические точки в настоящей работе мы рассматривать не будем, а ограничимся лишь типичными.

Из теоремы 1 следует, что через типичную параболическую точку $q$ проходит одна изотропная геодезическая, имеющая вид полукубической параболы с точкой возврата $q$ и касательным направлением $p_{0}=-\frac{b}{a}$. Найдем все остальные геодезические, входящие в точку $q$, которую без ограничения общности будем считать началом координат на плоскости $(t, x)$. Заметим, что из каждой эллиптической или гиперболической точки в каждом касательном направлении выходит ровно одна геодезическая, однако для параболической точки это не так. Действительно, из (6) или (7) следует, что через точку $\left(q, p_{*}\right)$ пространства $(t, x, p)$, где $\Delta(q)=0$ и $M\left(q, p_{*}\right) \neq 0$, проходит единственная траектория поля $(7)$ - вертикальная прямая, проекция которой на плоскость $(t, x)$ дает точку $q$, но не геодезическую. Таким образом, геодезические могут выходить из точки $q$ не во всех направлениях $p$, а только в таких, для которых $M(q, p)=0$. Это значит, что 1-графики геодезических входят только в особые точки поля

$$
\dot{t}=2 \Delta, \quad \dot{x}=2 p \Delta, \quad \dot{p}=M,
$$


которое получается из поля (7) умножением на $-2 F^{3 / 2}$. Компоненты поля (14) связаны функциональным соотношением $\dot{x}=p \dot{t}$, поэтому спектр линейной части поля (14) в любой особой точке $(q, p)$ всегда содержит одно нулевое значение. Как легко проверить, этот спектр равен $\left(\lambda_{1}, \lambda_{2}, 0\right)$, где

$$
\lambda_{1}=2\left(\Delta_{t}^{\prime}(q)+p \Delta_{x}^{\prime}(q)\right), \quad \lambda_{2}=M_{p}^{\prime}(q, p) .
$$

Исследуем особые точки поля (14), при этом нужно существенно различать два случая: $F(q, p) \neq 0$ и $F(q, p)=0$. Точки $(q, p)$ этих двух типов будем называть неизотропными и изотропными соответственно.

ЛЕмма 1. В любой неизотропной особой точке поля (14) имеет место резонанс

$$
\lambda_{1}+\lambda_{2}=0 .
$$

ДокАЗАтельство. Росток поля (14) в неизотропной точке получается умножением (7) на знакопостоянную функцию $-2 F^{3 / 2}$, следовательно, особая точка поля (14) будет особой и для поля (7), которое является частным случаем поля (4) для лагранжиана (2). Векторное поле (4) обладает легко проверяемым свойством: его дивергенция тождественно равна нулю (что отражает сохранение фазового объема для уравнения Эйлера-Лагранжа). Это свойство, вообще говоря, не сохраняется при умножении векторного поля $\vec{v}$ на гладкую скалярную функцию $\varphi$, однако в особых точках поля оно сохраняется. Действительно, из тождества

$$
\operatorname{div}(\varphi \vec{v}) \equiv \varphi \operatorname{div} \vec{v}+\langle\vec{v}, \nabla \varphi\rangle
$$

видно, что для любой гладкой $\varphi$ в точке, где $\vec{v}=0$ выполнено $\langle\vec{v}, \nabla \varphi\rangle=0$, и, следовательно, $\operatorname{div}(\varphi \vec{v})=\varphi \operatorname{div} \vec{v}$. Таким образом, в общих особых точках полей (14) и (7) дивергенция (т.е. след матрицы линейной части поля) равна нулю, что и дает соотношение (16).

Лемма 2. В типичной параболической точке $q$ для любого касательного направления р имеет место равенство

$$
M(q, p)=\frac{1}{3}\left(p-p_{0}\right) \widetilde{M}(q, p), \quad \text { где } \widetilde{M}(q, p)=2\left(\Delta_{t}^{\prime}(q)+p \Delta_{x}^{\prime}(q)\right)+M_{p}^{\prime}(q, p) .
$$

ДокАЗАТЕЛьство. Из теоремы 1 следует, что $M\left(q, p_{0}\right)=0$. Действительно, так как существует геодезическая (изотропная линия), входящая в точку $q$ с изотропным касательным направлением $p_{0}=-\frac{b}{a}$, то в силу сказанного выше это значит, что $\left(q, p_{0}\right)$ - особая точка поля (14). Следовательно, кубический многочлен $M(q, p)$ делится на $p-p_{0}$. Запишем это в виде равенства

$$
M(q, p)=\left(p-p_{0}\right) \sum_{i=0}^{2} \widetilde{\mu}_{i}(t, x) p^{i}
$$

с неопределенными коэффициентами $\widetilde{\mu}_{i}(t, x)$. Приравнивая коэффициенты при всех степенях $p$ в правых частях (8) и (19), получаем систему из четырех уравнений

$$
\widetilde{\mu}_{2}=\mu_{3}, \quad \widetilde{\mu}_{1}=p_{0} \mu_{3}+\mu_{2}, \quad \widetilde{\mu}_{0}=p_{0}^{2} \mu_{3}+p_{0} \mu_{2}+\mu_{1}, \quad p_{0} \widetilde{\mu}_{0}+\mu_{0}=0 .
$$


Для ее совместности нужно доказать равенство $p_{0}\left(p_{0}^{2} \mu_{3}+p_{0} \mu_{2}+\mu_{1}\right)+\mu_{0}=0$, которое равносильно $M\left(q, p_{0}\right)=0$ и поэтому верно. Остается доказать, что найденные коэффициенты $\widetilde{\mu}_{1}$ и $\widetilde{\mu}_{0}$ совпадают с соответствующими коэффициентами трехчлена $\frac{1}{3} \widetilde{M}(q, p)$ из $(18)$. Для этого нужно проверить, что в параболической точке $q$ выполнены равенства $\widetilde{\mu}_{1}=\frac{2}{3}\left(\mu_{2}+\Delta_{x}^{\prime}\right)$ и $\widetilde{\mu}_{0}=\frac{1}{3}\left(\mu_{1}+2 \Delta_{t}^{\prime}\right)$. Подставляя в них выражения $(20),(9)$ и $\Delta=b^{2}-a c$, с учетом того, что $\Delta(q)=0$ и $a \neq 0$, видим, что они выполнены.

Установленное леммой 2 разложение (18) кубического многочлена $M(q, p)$ дает второе доказательство утверждения леммы 1. Действительно, если $p-$ корень многочлена $M(q, p)$, то либо $p=p_{0}$ - изотропное направление, либо $p$ - корень многочлена $\widetilde{M}(q, p)$. Во втором случае из формул (15) и (18) сразу следует резонанс (16). Если же $p_{0}$ не является корнем $\widetilde{M}(q, p)$, то в изотропной особой точке $\left(q, p_{0}\right)$ соотношение $(16)$ не выполняется. Наконец, если $p_{0}$ является одновременно и корнем $\widetilde{M}(q, p)$, то $p_{0}$ - кратный корень многочлена $M(q, p)$, т.е. $M_{p}^{\prime}\left(q, p_{0}\right)=0$. Резонанс (16) в этом случае также имеет место, и в силу формулы (15) получаем, что $\lambda_{1}=\lambda_{2}=0$. Такой случай мы далее рассматривать не будет, т.е. всегда будем считать, что кубический многочлен $M(q, p)$ не имеет кратных корней.

Лемма 3. В типичной изотропной особой точке поля (14) имеет место резонанс

$$
\lambda_{1}=2 \lambda_{2}
$$

ДокАЗАТЕЛЬСтво. Отметим, что в изотропной особой точке неверны рассуждения, использованные нами при доказательстве леммы 1 для неизотропных точек. Действительно, в изотропной точке поле (7) не определено, т.е. в соотношении $(17)$, вообще говоря, $\langle\vec{v}, \nabla \varphi\rangle \neq 0$, и, следовательно, не выполнено равенство $\operatorname{div}(\varphi \vec{v})=\varphi \operatorname{div} \vec{v}$, приводящее к резонансному соотношению (16).

В изотропной точке $\left(q, p_{0}\right)$ с учетом $(15)$ и (9) получаем выражение

$$
\begin{aligned}
\lambda_{2}-\frac{1}{2} \lambda_{1} & =3 \mu_{3} p_{0}^{2}+\left(2 \mu_{2}-\Delta_{x}^{\prime}\right) p_{0}+\left(\mu_{1}-\Delta_{t}^{\prime}\right) \\
& =3 \mu_{3} p_{0}^{2}+3\left[2 b\left(a_{t}^{\prime}-b_{x}^{\prime}\right)+c a_{x}^{\prime}-a c_{x}^{\prime}\right] p_{0}+3\left[c a_{t}^{\prime}-b c_{x}^{\prime}\right]
\end{aligned}
$$

откуда с учетом $p_{0}=-\frac{b}{a}$ и опять же (9) получаем

$$
\frac{a^{2}}{3}\left(\lambda_{2}-\frac{1}{2} \lambda_{1}\right)=\mu_{3} b^{2}-a b\left[2 b\left(a_{t}^{\prime}-b_{x}^{\prime}\right)+c a_{x}^{\prime}-a c_{x}^{\prime}\right]+a^{2}\left[c a_{t}^{\prime}-b c_{x}^{\prime}\right]=\Delta\left(b a_{x}^{\prime}-a a_{t}^{\prime}\right) .
$$

В параболической точке $\Delta=0$, а в силу условия 1) в определении типичности имеем $a \neq 0$. Отсюда следует, что $\lambda_{2}=\frac{1}{2} \lambda_{1}$.

Теорема 2. Пусть $q-$ типичная параболическая точка, $\widetilde{p} \neq p_{0}-$ простой коренъ кубического многочлена $M(q, p)$. Тогда росток поля (14) в неизотропной особой точке $(q, \widetilde{p})$ гладко орбитально эквивалентен ростку

$$
\dot{\xi}=\xi, \quad \dot{\eta}=-\eta, \quad \dot{\zeta}=\xi \eta .
$$


ДокаЗАТЕЛЬСтво. Согласно леммам 1 и 2 в особой точке $(q, \widetilde{p})$ спектр линейной части поля (14) равен $(\lambda,-\lambda, 0)$, где $\lambda=2\left(\Delta_{t}^{\prime}(q)+\widetilde{p} \Delta_{x}^{\prime}(q)\right)$. Собственные направления имеют вид

$$
\vec{e}_{\lambda}=2 \lambda\left(\partial_{t}+\tilde{p} \partial_{x}\right)+\omega_{1} \partial_{p}, \quad \vec{e}_{-\lambda}=\partial_{p}, \quad \vec{e}_{0}=\lambda\left[\Delta_{x}^{\prime}(q) \partial_{t}-\Delta_{t}^{\prime}(q) \partial_{x}\right]+\omega_{2} \partial_{p},
$$

где $\omega_{1}=\left.\left(M_{t}^{\prime}+p M_{x}^{\prime}\right)\right|_{(q, \widetilde{p})}$ и $\omega_{2}=\left.\left(M_{t}^{\prime} \Delta_{x}^{\prime}-M_{x}^{\prime} \Delta_{t}^{\prime}\right)\right|_{(q, \widetilde{p})}$. Аффинная замена $(t, x, p) \rightarrow(\xi, \eta, \zeta)$ по формулам

$$
t=\lambda\left[2 \xi+\Delta_{x}^{\prime}(q) \zeta\right], \quad x=\lambda\left[2 \widetilde{p} \xi-\Delta_{t}^{\prime}(q) \zeta\right], \quad p-\widetilde{p}=\omega_{1} \xi+\eta+\omega_{2} \zeta,
$$

переводит точку $(q, \widetilde{p})$ в начало координат пространства $(\xi, \eta, \zeta)$ и приводит поле (14) к виду

$$
\dot{\xi}=\lambda \xi+\cdots, \quad \dot{\eta}=-\lambda \eta+\cdots, \quad \dot{\zeta}=0+\cdots,
$$

где многоточия обозначают члены выше первой степени. Заметим, что компоненты поля (14) принадлежат идеалу, порожденному двумя из них (компонентами при $\dot{t}$ и $\dot{p})$. Это свойство, как легко проверить, инвариантно относительно замен переменных, поэтому компоненты поля (24) также принадлежат идеалу, порожденному двумя из них. Очевидно, что в качестве таких образующих могут быть выбраны только компоненты в двух первых уравнениях. Таким образом, множество особых точек (оно же - центральное многообразие $W^{c}$ ) поля (24) задается уравнениями $\dot{\xi}=0$ и $\dot{\eta}=0$.

Покажем, что третья компонента поля (24) содержит резонансный моном $\xi \eta$ с отличным от нуля коэффициентом. Из (23) получаем выражение $\frac{1}{2} \lambda^{2} \zeta=$ $\widetilde{p} t-x$, откуда

$$
\begin{aligned}
\frac{1}{4} \lambda^{2} \dot{\zeta} & =\frac{1}{2}(\widetilde{p} \dot{t}-\dot{x})=(\widetilde{p}-p) \Delta(t, x)=(\widetilde{p}-p)\left[\Delta_{t}^{\prime}(q) t+\Delta_{x}^{\prime}(q) x+\cdots\right] \\
& =-\left(\omega_{1} \xi+\eta+\omega_{2} \zeta\right) \lambda\left[\Delta_{t}^{\prime}(q)\left(2 \xi+\Delta_{x}^{\prime}(q) \zeta\right)+\Delta_{x}^{\prime}(q)\left(2 \widetilde{p} \xi+\Delta_{t}^{\prime}(q) \zeta\right)+\cdots\right] \\
& =-\lambda\left(\omega_{1} \xi+\eta+\omega_{2} \zeta\right)\left[2\left(\Delta_{t}^{\prime}(q)+\widetilde{p} \Delta_{x}^{\prime}(q)\right) \xi+\cdots\right] \\
& =-\lambda^{2}\left(\omega_{1} \xi+\eta+\omega_{2} \zeta\right)[\xi+\cdots] .
\end{aligned}
$$

Следовательно, компонента $\dot{\zeta}$ содержит член $-4 \xi \eta$. Росток такого поля орбитально эквивалентен нормальной форме (22). Для конечно гладкой (т.е. с любой наперед заданной степенью гладкости) эквивалентности это сразу следует из [16; теорема 6$]$. Для $C^{\infty}$-гладкой эквивалентности это следует из [17; теорема 22].

Поле (22) имеет первый интеграл $U=\xi \eta$ и, следовательно, инвариантное слоение $\xi \eta=$ const с особенностью вдоль оси $\zeta$, которая целиком состоит из особых точек поля (22) и является его центральным многообразием. Оси $\xi$ и $\eta$ являются одномерными неустойчивым и устойчивым многообразиями, входящими в начало координат. Аналогично, в каждую точку оси $\zeta$ входят две траектории (22) - прямые, параллельные осям $\xi$ и $\eta$, все остальные трактории являются седловыми. Переходя к исходному полю (14), мы заключаем, что оно имеет ровно две траектории, входящие в особую точку $(q, \widetilde{p})$. Они являются соответственно неустойчивым и устойчивым инвариантными многообразиями 
поля (14) и имеют касательные направления $\vec{e}_{\lambda}$ и $\vec{e}_{-\lambda}$. Так как направление $\vec{e}_{-\lambda}$ вертикально и первые две компоненты поля (14) обращаются в нуль в точке $q$ тождественно по $p$, то соответствующая направлению $\vec{e}_{-\lambda}$ траектория - это вертикальная прямая в пространстве $(t, x, p)$, и ее проекция на плоскость $(t, x)$ дает точку $q$, а не геодезическую. Остается лишь вторая траектория, входящая в особую точку $(q, \widetilde{p})$ с невертикальным касательным направлением $\vec{e}_{\lambda}$. Ее проекция на плоскость $(t, x)$ дает гладкую геодезическую с 1 -струей $x(t)=\widetilde{p} t$.

Теорема 3. Пустъ $q$ - типичная параболическая точка, $\widetilde{M}\left(q, p_{0}\right) \neq 0$. Тогда росток поля (14) в изотропной особой точке $\left(q, p_{0}\right)$ гладко орбитально эквивалентен ростку

$$
\dot{\xi}=2 \xi, \quad \dot{\eta}=\eta, \quad \dot{\zeta}=0
$$

ДокАЗАтельство. Согласно леммам 2 и 3 в особой точке $\left(q, p_{0}\right)$ спектр линейной части поля (14) равен $(2 \lambda, \lambda, 0)$, где $\lambda=\Delta_{t}^{\prime}(q)+p_{0} \Delta_{x}^{\prime}(q)$. При этом собственные направления имеют вид

$$
\vec{e}_{2 \lambda}=\lambda\left(\partial_{t}+p_{0} \partial_{x}\right)+\omega_{1} \partial_{p}, \quad \vec{e}_{\lambda}=\partial_{p}, \quad \vec{e}_{0}=\lambda\left[\Delta_{x}^{\prime}(q) \partial_{t}-\Delta_{t}^{\prime}(q) \partial_{x}\right]-\omega_{2} \partial_{p}
$$

где $\omega_{1}=\left.\left(M_{t}^{\prime}+p M_{x}^{\prime}\right)\right|_{\left(q, p_{0}\right)}$ и $\omega_{2}=\left.\left(M_{t}^{\prime} \Delta_{x}^{\prime}-M_{x}^{\prime} \Delta_{t}^{\prime}\right)\right|_{\left(q, p_{0}\right)}$. Аффинная замена $(t, x, p) \rightarrow(\xi, \eta, \zeta)$ по формуле

$$
t=\lambda\left[\xi+\Delta_{x}^{\prime}(q) \zeta\right], \quad x=\lambda\left[p_{0} \xi-\Delta_{t}^{\prime}(q) \zeta\right], \quad p-p_{0}=\omega_{1} \xi+\eta-\omega_{2} \zeta,
$$

переводит точку $\left(q, p_{0}\right)$ в начало координат пространства $(\xi, \eta, \zeta)$ и приводит поле (14) к виду

$$
\dot{\xi}=2 \lambda \xi+\cdots, \quad \dot{\eta}=\lambda \eta+\cdots, \quad \dot{\zeta}=0+\cdots,
$$

где многоточия обозначают члены выше первой степени. Компоненты поля (28) принадлежат идеалу, порожденному первыми двумя из них. Росток такого поля орбитально эквивалентен нормальной форме

$$
\dot{\xi}=2 \xi+\psi(\zeta) \eta^{2}, \quad \dot{\eta}=\eta, \quad \dot{\zeta}=0
$$

с некоторой гладкой функцией $\psi(\zeta)$. Для конечно гладкой (т.е. с любой наперед заданной степенью гладкости) эквивалентности это следует из [16; теорема 2]. Нетрудно показать, что здесь имеет место и $C^{\infty}$-гладкая эквивалентность (например, с помощью метода, использованного при доказательстве теоремы 20 в [17]). Более того, в случае, когда поле (14) аналитическое, имеет место аналитическая эквивалентность нормальной форме (29), для этого можно использовать способ доказательства сходимости нормализующей замены, используемый для ростка поля со спектром в области Пуанкаре (см. [12], [13], [18], [19], а также [20], [21]).

Теперь докажем, что $\psi(\zeta) \equiv 0$ в нормальной форме $(29)$.

1. Покажем, что в первом уравнении системы (28) коэффициент при мономе $\eta^{2}$ равен нулю. Из (27) получаем выражение $\lambda^{2} \xi=\Delta_{t}^{\prime}(q) t+\Delta_{x}^{\prime}(q) x$, откуда, выделяя в $\Delta(t, x)$ линейные члены и производя замену $(27)$, с учетом равенства 
$\lambda=\Delta_{t}^{\prime}(q)+p_{0} \Delta_{x}^{\prime}(q)$ получаем

$$
\begin{aligned}
\frac{1}{2} \lambda^{2} \dot{\xi}= & \frac{1}{2}\left[\Delta_{t}^{\prime}(q) \dot{t}+\Delta_{x}^{\prime}(q) \dot{x}\right]=\Delta(t, x)\left[\Delta_{t}^{\prime}(q)+p \Delta_{x}^{\prime}(q)\right] \\
= & \lambda\left[\Delta_{t}^{\prime}(q)\left(\xi+\Delta_{x}^{\prime}(q) \zeta\right)+\Delta_{x}^{\prime}(q)\left(p_{0} \xi-\Delta_{t}^{\prime}(q) \zeta\right)+R(\xi, \zeta)+\cdots\right] \\
& \times\left[\Delta_{t}^{\prime}(q)+\left(p_{0}+\omega_{1} \xi+\eta-\omega_{2} \zeta\right) \Delta_{x}^{\prime}(q)\right] \\
= & \lambda[\lambda \xi+R(\xi, \zeta)+\cdots]\left[\lambda+\Delta_{x}^{\prime}(q)\left(\omega_{1} \xi+\eta-\omega_{2} \zeta\right)\right]
\end{aligned}
$$

где $R(\xi, \zeta)$ - однородный многочлен второй степени, а многоточия обозначают члены выше второй степени. Отсюда получаем равенство

$$
\dot{\xi}=2 \lambda \xi+2\left[\Delta_{x}^{\prime}(q) \xi\left(\omega_{1} \xi+\eta-\omega_{2} \zeta\right)+R(\xi, \zeta)\right]+\cdots,
$$

показывающее, что первое уравнение системы (28) не содержит моном $\eta^{2}$.

2. Покажем, что если в системе (29) сделать замену переменных с тождественной линейной частью, то коэффициент при мономе $\eta^{2}$ в ее первом уравнении не изменится. Такую замену можно представить как композицию трех замен, в каждой из которых меняется только одна переменная:

$$
\xi \rightarrow \xi+f(\xi, \eta, \zeta), \quad \eta \rightarrow \eta+g(\xi, \eta, \zeta), \quad \zeta \rightarrow \zeta+h(\xi, \eta, \zeta),
$$

функции $f, g, h$ содержат члены выше первой степени. Очевидно, что две последние замены не меняют коэффициент при $\eta^{2}$ в первом уравнении системы (29), и остается рассмотреть только первую замену (30). Пусть она имеет вид

$$
\Xi=\xi+\sum_{i+j+k=2} \alpha_{i j k} \xi^{i} \eta^{j} \zeta^{k}+\cdots, \quad \xi=\Xi+\sum_{i+j+k=2} \beta_{i j k} \Xi^{i} \eta^{j} \zeta^{k}+\cdots,
$$

где индексы $i, j, k$ принимают целые неотрицательные значения, а многоточия обозначают члены выше второй степени. Подставляя выражение для $\xi$ в выражение для $\Xi$ (или наоборот) и приводя подобные члены, получаем равенства $\alpha_{i j k}+\beta_{i j k}=0$. Пусть первое уравнение системы (29) имеет вид

$$
\dot{\xi}=2 \xi+\sum_{i+j+k=2} \gamma_{i j k} \xi^{i} \eta^{j} \zeta^{k}+\cdots
$$

где многоточие обозначает члены выше второй степени. После замены (31) оно перейдет в уравнение

$$
\begin{aligned}
\dot{\Xi}= & \dot{\xi}\left(1+2 \alpha_{200} \xi+\alpha_{110} \eta+\alpha_{011} \zeta\right)+\dot{\eta}\left(\alpha_{110} \xi+2 \alpha_{020} \eta+\alpha_{011} \zeta\right) \\
& +\dot{\zeta}\left(\alpha_{101} \xi+\alpha_{011} \eta+2 \alpha_{002} \zeta\right)+\cdots \\
= & \left(2 \xi+\sum_{i+j+k=2} \gamma_{i j k} \xi^{i} \eta^{j} \zeta^{k}\right)\left(1+2 \alpha_{200} \xi+\alpha_{110} \eta+\alpha_{011} \zeta\right) \\
& +\eta\left(\alpha_{110} \xi+2 \alpha_{020} \eta+\alpha_{011} \zeta\right)+\cdots \\
= & \left(2 \Xi+\sum_{i+j+k=2}\left(2 \beta_{i j k}+\gamma_{i j k}\right) \Xi^{i} \eta^{j} \zeta^{k}\right)\left(1+2 \alpha_{200} \Xi+\alpha_{110} \eta+\alpha_{011} \zeta\right) \\
& +\eta\left(\alpha_{110} \Xi+2 \alpha_{020} \eta+\alpha_{011} \zeta\right)+\cdots .
\end{aligned}
$$


Квадратичная часть уравнения (33) содержит моном $\left(2 \alpha_{020}+2 \beta_{020}+\gamma_{020}\right) \eta^{2}$, т.е. с учетом соотношений $\alpha_{i j k}+\beta_{i j k}=0$ моном $\gamma_{020} \eta^{2}$. Таким образом, коэффициенты при $\eta^{2}$ в уравнениях (32) и (33) совпадают.

3. Росток поля (14) приводится к орбитальной нормальной форме (29) с помощью некоторой замены координат в фазовом пространстве и умножения поля на знакопостоянную функцию $\varphi$. При этом замена координат может быть осуществлена как композиция линейного преобразования, нормализующего линейную часть поля (14), т.е. приводящего его к форме (28), и ростка диффеоморфизма с линейной частью, приводящего поле (28) к виду, из которого нормальная форма (29) получается умножением на знакопостоянную функцию $\varphi$. Из доказанного в пп. 1, 2 следует, что коэффициент при мономе $\eta^{2}$ в первом уравнении системы (29) будет равен нулю, т.е. $\psi(0)=0$. Заметим, что все наши рассуждения справедливы для любой типичной параболической точки $q$ такой, что $\widetilde{M}\left(q, p_{0}\right) \neq 0$. Очевидно, что все параболические точки, достаточно близкие к $q$, удовлетворяют этим условиям. С другой стороны, приведение поля (14) к нормальной форме (29) имеет место в некоторой окрестности точки $\left(q, p_{0}\right)$, поэтому имеет место равенство $\psi(\zeta)=0$ для всех $\zeta$, достаточно малых по модулю.

Поле (25) имеет первый интеграл $U=\zeta$ и, следовательно, гладкое инвариантное слоение $\zeta=$ const. Ось $\zeta$ целиком состоит из особых точек поля (25) и является его центральным многообразием. Из (27) получаем $\lambda^{2} \zeta=p_{0} t-x$, и, соответственно, исходное поле (14) имеет первый интеграл $U=p_{0} t-x+\cdots$, где многоточие означает члены выше первой степени. Центральное многообразие поля (14) состоит из его особых точек и задается уравнениями $\Delta=0$ и $M=0$, оно трансверсально инвариантному слоению $U=$ const. Заметим, что слои $U=p_{0} t-x+\cdots=$ const трансверсальны поверхности $\mathscr{F}$, задаваемой уравнением $F(t, x, p)=0$. Действительно, их пересечению в точке $\left(q, p_{0}\right)$ соответствует матрица

$$
\left(\begin{array}{ccc}
F_{t}^{\prime} & F_{x}^{\prime} & F_{p}^{\prime} \\
p & -1 & 0
\end{array}\right) .
$$

В точке $\left(q, p_{0}\right)$ производная $F_{p}^{\prime}$ равна нулю, но минор, стоящий в двух первых столбцах матрицы, не равен нулю: $F_{t}^{\prime}+p F_{x}^{\prime} \neq 0$ (см. формулу (13)). Следовательно, поверхность $\mathscr{F}$ пересекается со слоем $U=$ const, проходящим через точку $\left(q, p_{0}\right)$, по гладкой кривой $\gamma_{0}^{\prime}$, которая согласно теореме 1 является траекторией обеих систем (6) и (11), т.е. 1-графиком изотропной геодезической $\gamma_{0}$, проходящей через точку $q .^{4}$

Теперь рассмотрим фазовый портрет ограничения поля (14) на инвариантный слой $U=$ const, проходящий через особую точку $\left(q, p_{0}\right)$. Так как первый интеграл определен с точностью до прибавления константы, то без ограничения общности можно считать далее, что это слой $U=0$. В координатах $(\xi, \eta, \zeta)$ ему соответствует ограничение нормальной формы (25) на слой $\zeta=0$. Оно дает узел $\frac{d \xi}{d \eta}=\frac{2 \xi}{\eta}$, траекториями которого являются, во-первых, ось $\xi$ и, во-вторых, семейство кривых

$$
\xi=\alpha \eta^{2}, \quad \zeta=0, \quad \alpha \in \mathbb{R} .
$$

\footnotetext{
43десь и далее будем использовать следующее обозначение: если $\gamma$ - кривая на плоскости $(t, x)$, то через $\gamma^{\prime}$ обозначается ее 1 -график в пространстве $(t, x, p)$.
} 
Траекториям поля (25) соответствуют траектории поля (14), получающиеся из первых под действием диффеоморфизма $f$ с аффинной частью (27). Выбрав на инвариантном слое $U=0$ локальные координаты $(t, p)$, ограничение $f$ на $U=0$ можно записать в виде отображения $(\xi, \eta) \rightarrow(t, p)$, заданного формулой

$$
t=\lambda \xi+\sum_{i+j=2} a_{i j} \xi^{i} \eta^{j}+\cdots, \quad p-p_{0}=\omega_{1} \xi+\eta+\sum_{i+j=2} b_{i j} \xi^{i} \eta^{j}+\cdots
$$

где $a_{i j}$ и $b_{i j}$ - некоторые коэффициенты, а многоточия обозначают мономы от переменных $\xi$ и $\eta$ выше второй степени. Подставляя в (35) траектории поля (25), лежащие на плоскости $\zeta=0$, мы получим траектории поля (14), лежащие на слое $U=0$. Так, оси $\xi$ соответствует траектория $\gamma_{\infty}^{\prime}$, заданная формулой

$$
t=\lambda \xi+a_{20} \xi^{2}+o\left(\xi^{2}\right), \quad p-p_{0}=\omega_{1} \xi+b_{20} \xi^{2}+o\left(\xi^{2}\right)
$$

с параметром $\xi$. Как видно из $(36)$, кривая $\gamma_{\infty}^{\prime}$ в точке $\left(q, p_{0}\right)$ имеет касательное направление $d p: d t=\omega_{1}: \lambda$. Следовательно, она не вертикальна, и ее проекция $\gamma_{\infty}$ на плоскость $(t, x)$ вдоль $p$ дает гладкую геодезическую с 2-струей $x(t)=p_{0} t+\omega_{1} t^{2} /(2 \lambda)$.

Кривым семейства (34) соответствуют траектории $\gamma_{\alpha}^{\prime}$, заданные формулой

$$
t=\left(\alpha \lambda+a_{02}\right) \eta^{2}+o\left(\eta^{2}\right), \quad p-p_{0}=\eta+\left(\alpha \omega_{1}+b_{02}\right) \eta^{2}+o\left(\eta^{2}\right)
$$

с параметром $\eta$, лежащие в полуплоскости $t \geqslant 0$ при $\alpha \lambda+a_{02}>0$ и в полуплоскости $t \leqslant 0$ при $\alpha \lambda+a_{02}<0$. Как видно из $(37)$, все кривые $\gamma_{\alpha}^{\prime}$ в точке $\left(q, p_{0}\right)$ имеют касательное направление $d p: d t=1: 0$, причем

$$
\frac{d t}{d p}=2\left(\alpha \lambda+a_{02}\right) \eta+o(\eta), \quad \frac{d^{2} t}{d p^{2}}=2\left(\alpha \lambda+a_{02}\right)+o(1)
$$

откуда видно, что при всех таких $\alpha$, для которых $\alpha \lambda+a_{02} \neq 0$, кривые $\gamma_{\alpha}^{\prime}$ имеют с осью $p$ касание второго порядка, а в случае $\lambda \alpha_{*}+a_{02}=0$ кривая $\gamma_{\alpha_{*}}^{\prime}$ имеет с осью $p$ касание выше второго порядка (как легко показать, бесконечного). Так как среди траекторий поля $(14)$, входящих в особую точку $\left(q, p_{0}\right)$, имеется вертикальная прямая (параллельная оси $p$ ), то именно она и совпадает с кривой $\gamma_{\alpha_{*}}^{\prime}$. Таким образом, проекция траектории $\gamma_{\alpha_{*}}^{\prime}$ на плоскость $(t, x)$ вдоль $p$ дает точку $q$, но не геодезическую, а проекции $\gamma_{\alpha}^{\prime}$ при $\alpha \neq \alpha_{*}$ дают геодезические $\gamma_{\alpha}$, имеющие в точке $q$ особенность - полукубическую точку возврата (это следует из формулы (38)) с общим касательным направлением $d x: d t=p_{0}$. Заметим, что при $\alpha \rightarrow \infty$ парабола (34) стремится к оси $\xi$ (точнее, к одной из полуосей в зависимости от знака $\alpha$ ). Соответственно диффеоморфная полукубической параболе геодезическая $\gamma_{\alpha}, \alpha \neq \alpha_{*}$, стремится при $\alpha \rightarrow \infty$ к гладкой геодезической $\gamma_{\infty}$ (точнее, к одной из ее половинок в зависимости от знака $\alpha$ ). Таким образом, естественно включить геодезическую $\gamma_{\infty}$ в семейство $\gamma_{\alpha}$ (при значении параметра $\alpha=\infty)$ и исключить из этого семейства $\gamma_{\alpha_{*}}$. Мы сделаем это, определив множество индексов $\mathfrak{A}=\mathbb{R} \backslash\left\{\alpha_{*}\right\} \cup\{\infty\}$, и далее будем рассматривать геодезические семейства $\gamma_{\alpha}, \alpha \in \mathfrak{A}$. 


\section{§ 3. Экстремальные свойства геодезических}

Разделим входящие в точку $q$ геодезические семейства $\gamma_{\alpha}, \alpha \in \mathfrak{A}$, на три типа: времениподобные $(F>0)$, пространственноподобные $(F<0)$ и изотропные $(F=0) .{ }^{5}$ В семействе $\gamma_{\alpha}, \alpha \in \mathfrak{A}$, имеется единственная изотропная геодезическая, которую мы обозначили выше через $\gamma_{0}$. Для удобства мы будем далее считать, что она является кривой $\gamma_{\alpha}$ при $\alpha=0$ (это достигается заменой параметра $\left.\alpha \rightarrow \alpha+\alpha_{0}\right)$. Траектория $\gamma_{0}^{\prime}$ разделяет инвариантный слой $U=0$ на две части: $F>0$ и $F<0$, и любая другая траектория $\gamma_{\alpha}^{\prime}, \alpha \in \mathfrak{A}$, целиком содержится в одной из этих частей, за исключением общей для всех траекторий точки $\left(q, p_{0}\right)$. Таким образом, изотропная геодезическая $\gamma_{0}$ разделяет семейство $\gamma_{\alpha}, \alpha \in \mathfrak{A}$, на две части: $\alpha>0$ и $\alpha<0$, состоящие из времениподобных и пространственноподобных геодезических.

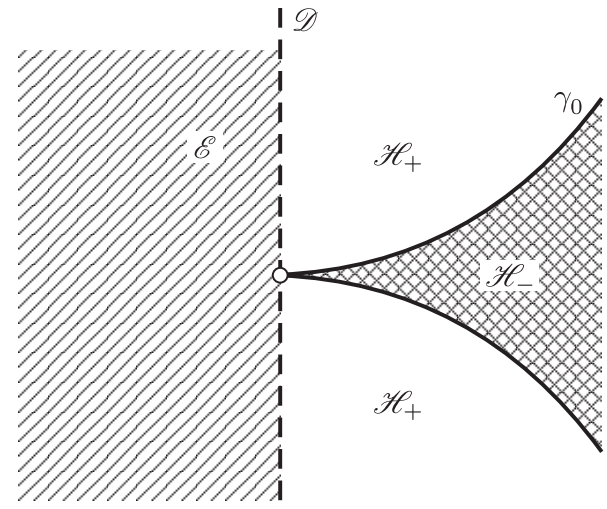

Рис. 1

Напомним, что в окрестности типичной параболической точки $q$ дискриминантная кривая $\mathscr{D}$ разбивает плоскость $(t, x)$ на эллиптическую $\mathscr{E}(\Delta<0)$ и гиперболическую области $\mathscr{H}(\Delta>0)$. Изотропная геодезическая $\gamma_{0}$ лежит в области $\mathscr{H}$ и делит ее на две части: $\mathscr{H}_{+}$, примыкающую к $\mathscr{E}$, и сектор $\mathscr{H}_{-}$, лежащий между ветвей полукубической параболы $\gamma_{0}$ (рис. 1). Предположим для определенности, что $a>0$ (случай $a<0$ рассматривается аналогично). Тогда все времениподобные геодезические семейства $\gamma_{\alpha}, \alpha \in \mathfrak{A}$, лежат в $\mathscr{E} \cup \mathscr{H}_{+}$, а все пространственноподобные - в области $\mathscr{H}_{-}$(рис. 2).

Рассмотрим случай общего положения: пусть $q$ - типичная параболическая точка, в которой старший коэффициент $\mu_{3}$ кубического многочлена $M(q, p)$ и дискриминант $\widetilde{D}(q)$ квадратного трехчлена $\widetilde{M}(q, p)$ не равны нулю. Тогда возможны две ситуации: $\widetilde{D}<0$, и многочлен $M(q, p)$ имеет единственный корень $p_{0}$, который удовлетворяет условию $\widetilde{M}\left(q, p_{0}\right) \neq 0$; и $\widetilde{D}>0$, и $M(q, p)$ имеет три различных корня $p_{0}, \widetilde{p}_{1}, \widetilde{p}_{2}$, где $\widetilde{M}\left(q, \widetilde{p}_{i}\right)=0$. В обоих случаях теорема 3

\footnotetext{
53десь имеется в виду, что неравенства выполняются во всех точках геодезической, достаточно близких к $q$, но за исключением ее самой, так как в точке $q$ все геодезические этого семейства имеют изотропное касательное направление $p_{0}=-b / a$.
} 


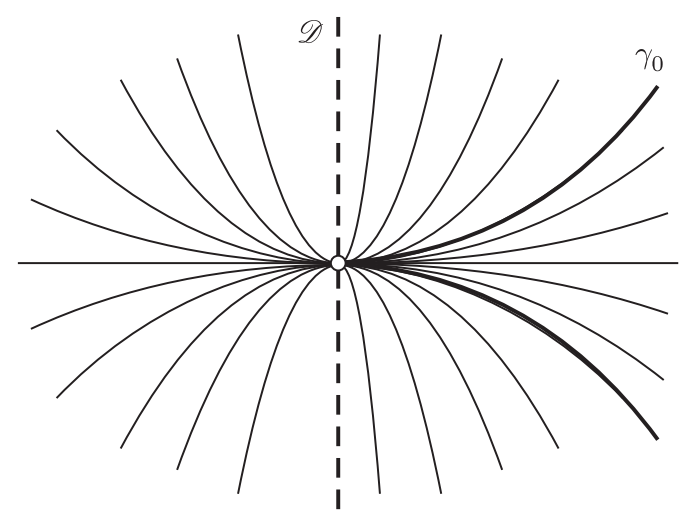

Рис. 2

дает описанное выше семейство геодезических $\gamma_{\alpha}, \alpha \in \mathfrak{A}$, касающихся изотропного направления $p_{0}$.

В первом случае $(\widetilde{D}<0)$ это и есть все геодезические, входящие в точку $q$ с определенным направлением касательной. Так как $p_{0}-$ единственный корень многочлена $M(q, p)$, то для этого нужно доказать лишь отсутствие геодезических с касательным направлением $p=\infty$, т.е. $d x: d t=1: 0$, которое мы ранее исключили из рассмотрения, выбрав координату $t$ в качестве независимой переменной, а координату $x$ - в качестве зависимой, хотя геометрически обе координаты совершенно равноправны. Поменяв $t$ и $x$ местами, мы видим, что в формулах (1) и (2) поменяются местами коэффициенты $a$ и $c$. Записав уравнение Эйлера-Лагранжа для лагранжиана (2) в новых переменных, мы получим поле $(7)$, в котором $\Delta$ и $F$ те же самые, а многочлен $M=\sum_{i=0}^{3} \mu_{i}(t, x) p^{i}$ заменится на $N=\sum_{i=0}^{3} \nu_{i}(t, x) p^{i}$, где $\nu_{i}+\mu_{3-i}=0, i=0, \ldots, 3$. Касательному направлению $d x: d t=1: 0$ в старых переменных соответствует значение $p=\infty$, а в новых - значение $p=0$. Таким образом, для существования геодезических с этим направлением необходимо, чтобы многочлен $N(q, p)$ имел корень $p=0$, что невозможно в силу условия $\nu_{0}=\mu_{3} \neq 0$.

Во втором случае $(\widetilde{D}>0)$ отсутствие геодезических с касательным направлением $d x: d t=1: 0$ устанавливается точно так же. Однако многочлен $M(q, p)$ имеет еще два простые корня $\widetilde{p}_{1}$ и $\widetilde{p}_{2}$, которым согласно теореме 2 соответствуют гладкие геодезические $\widetilde{\gamma}_{1}$ и $\widetilde{\gamma}_{2}$, входящие в точку $q$ с касательными направлениями $\widetilde{p}_{1}$ и $\widetilde{p}_{2}$, трансверсальными дискриминантной кривой $\mathscr{D}$. Так как в точке $q$ дискриминант $\Delta$ квадратного трехчлена $F=a p^{2}+2 b p+c$ равен нулю, то он представляет собой полный квадрат $F=a\left(p-p_{0}\right)^{2}$, и для всех $p$ выполнено или неравенство $F \geqslant 0$ (если $a>0$ ), или неравенство $F \leqslant 0$ (если $a<0)$. Следовательно, оба направления $\widetilde{p}_{1}$ и $\widetilde{p}_{2}$ являются времениподобными при $a>0$ и пространственноподобными при $a<0$.

Рассмотрим все проходящие через $q$ геодезические с точки зрения экстремальных свойств функционала длины в метрике (1), т.е.

$$
J(\gamma)=\int_{t_{0}}^{t_{1}} \sqrt{F\left(\gamma^{\prime}\right)} d t .
$$


В случае $a>0$ в эллиптической области $\mathscr{E}$ метрика $Q$ риманова и лежащие в $\mathscr{E}$ геодезические доставляют локальный минимум функционала (39); см., например, [22; гл.7]. В случае $a<0$ все кривые в области $\mathscr{E}$ имеют чисто мнимую длину, и, вынося $i$ из-под корня в формуле (39), мы получаем, что лежащие в $\mathscr{E}$ геодезические доставляют локальный минимум мнимой части функционала (39). Рассмотрим теперь области $\mathscr{H}_{+}$и $\mathscr{H}_{-}$, предполагая, что $a>0$ (в случае $a<0$ рассуждения аналогичны). Все геодезические в $\mathscr{H}_{+}$имеют вещественную длину, а в $\mathscr{H}_{-}-$чисто мнимую, но длина $J(\gamma)$ произвольной кривой $\gamma$ в обеих областях является, вообще говоря, комплексной.

Обозначим через $J_{\mathrm{R}}$ и $J_{\mathrm{I}}$ соответственно вещественную и мнимую части функционала (39), т.е.

$$
J(\gamma)=J_{\mathrm{R}}(\gamma)+i J_{\mathrm{I}}(\gamma), \quad J_{\mathrm{R}}(\gamma) \geqslant 0, \quad J_{\mathrm{I}}(\gamma) \geqslant 0,
$$

а также введем условные обозначения: запись $\min (\max )$ для $J_{\mathrm{R}}(\gamma)$ означает, что функционал $J_{\mathrm{R}}(\gamma)$ достигает на кривой $\gamma$ локального минимума (максимума) в определенном ниже смысле. Пусть геодезическая $\gamma$ выходит из параболической точки $q$ при $t=0$ и содержится в какой-либо из областей $\mathscr{E}, \mathscr{H}_{+}, \mathscr{H}_{-}$ при $t>0$. Тогда $\min (\max )$ для $J_{\mathrm{R}}(\gamma)$ означает, что для любой точки $t_{0}>0$ найдется такая точка $t_{1}>t_{0}$, что для произвольной кривой $\omega$, соединяющей те же точки, что и дуга $\gamma$ на $\left[t_{0}, t_{1}\right]$, выполнено неравенство $J_{\mathrm{R}}(\gamma) \leqslant J_{\mathrm{R}}(\omega)$ $\left(J_{\mathrm{R}}(\gamma) \geqslant J_{\mathrm{R}}(\omega)\right)$. Запись $\min (\max )$ для $J_{\mathrm{I}}(\gamma)$ определяется аналогично.

Теорема 4. В случае $а>0$ экстремальные свойства геодезических описъваются следующей таблищей:

\begin{tabular}{|c|c|c|c|c|}
\hline & $\gamma \subset \mathscr{E}$ & $\gamma \subset \mathscr{H}_{+}$ & $\gamma=\gamma_{0}$ & $\gamma \subset \mathscr{H}_{-}$ \\
\hline$J_{\mathrm{R}}(\gamma)$ & $\min >0$ & $\max >0$ & 0 & 0 \\
\hline$J_{\mathrm{I}}(\gamma)$ & 0 & 0 & 0 & $\max >0$ \\
\hline
\end{tabular}

Здесь $\gamma$ - дуги геодезических $\gamma_{\alpha}, \alpha \in \mathfrak{A}$, или $\widetilde{\gamma}_{1,2}$, иеликом лежашие в одной из открытых областей $\mathscr{E}, \mathscr{H}_{+}, \mathscr{H}_{-}$, либо дуга изотропной геодезической $\gamma_{0}$, разделяющей области $\mathscr{H}_{+}$и $\mathscr{H}_{-}$вещественной и мнимой длинъ.

ЗАмЕчАниЕ. Для случая $a<0$ аналогичная таблица получается, если переставить вещественную и мнимую части $\left(J_{\mathrm{R}}\right.$ и $\left.J_{\mathrm{I}}\right)$ местами.

ДоКАЗАТЕЛЬСТВо ТЕОРЕмЫ 4. Первый и третий случаи таблицы $(\gamma \subset \mathscr{E}$ и $\left.\gamma=\gamma_{0}\right)$ очевидны. Пусть $\gamma \subset \mathscr{H}_{+}-$дуга геодезической, соответствующая даному выше определению, и $\omega$ - произвольная кривая, соединяющая те же точки, что и дуга $\gamma$ на отрезке $\left[t_{0}, t_{1}\right]$. Функция $F\left(\gamma^{\prime}\right)$ на $\left[t_{0}, t_{1}\right]$ положительна, но функция $F\left(\omega^{\prime}\right)$ может иметь разные знаки даже в том случае, если кривая $\omega$ целиком лежит в области $\mathscr{H}_{+}$(отметим, что в определении $\min (\max )$ условия $\omega \subset \mathscr{H}_{+}$нет).

Покажем сначала, что если $t_{1}-t_{0}$ достаточно мало, то для всех $\omega$, для которых $F\left(\omega^{\prime}\right) \geqslant 0$ при $t \in\left[t_{0}, t_{1}\right]$, выполнено неравенство $J(\omega) \leqslant J(\gamma)$. Для этого воспользуемся условиями отрицательной определенности второй вариации (см. [23]). Так как $\gamma \subset \mathscr{H}_{+}$, то вдоль $\gamma^{\prime}$ имеем $\Delta>0$ и $F>0$, откуда с учетом (5) получаем $L_{p p}^{\prime \prime}<0$. Таким образом, для доказательства отрицательной определенности функционала $\delta^{2} J$ нам достаточно проверить усиленное 
условие Якоби - отсутствие сопряженных точек, т.е. обращающихся в нуль на $\left(t_{0}, t_{1}\right]$ нетривиальных решений $h(t)$ задачи Коши

$$
\frac{d}{d t}\left(A(t) h^{\prime}+C(t) h\right)-\left(C(t) h^{\prime}+B(t) h\right)=0, \quad h\left(t_{0}\right)=0,
$$

где $A(t)=L_{p p}^{\prime \prime}\left(\gamma^{\prime}\right), B(t)=L_{x x}^{\prime \prime}\left(\gamma^{\prime}\right)$ и $C(t)=L_{x p}^{\prime \prime}\left(\gamma^{\prime}\right)$. На отрезке $\left[t_{0}, t_{1}\right]$ функция $A^{\prime}(t)$ не обращается в нуль, и дифференциальное уравнение в задаче (40) имеет вид $h^{\prime \prime}+\alpha(t) h^{\prime}+\beta(t) h=0$ с гладкими коэффициентами $\alpha(t)$ и $\beta(t)$. Общее решение такого уравнения представимо в виде линейной комбинации любых двух его независимых решений, следовательно, общее решение задачи (40) имеет вид $h(t)=c \chi(t)$, где $c$ - произвольная постоянная и $\chi(t)$ - любое нетривиальное решение этого уравнения, удовлетворяющее условию $\chi\left(t_{0}\right)=0$. Таким образом, все решения задачи (40) обращаются на $\left(t_{0}, t_{1}\right]$ в нуль в той и только той точке $t_{*}$, где $\chi\left(t_{*}\right)=0$ (если такая точка $t_{*}$ вообще существует). Выбрав $t_{1}<t_{*}$, мы получим, что на $\left(t_{0}, t_{1}\right]$ нет сопряженных точек и выполнено достаточное условие отрицательной определенности $\delta^{2} J$ (см. [23]).

Пусть $\left[t_{0}, t_{1}\right]$ - построенный выше отрезок, $\left[\tau_{0}, \tau_{1}\right] \subset\left[t_{0}, t_{1}\right]$ и $I(\gamma)$ - функционал длины, определенный формулой (39) с интегралом по отрезку $\left[\tau_{0}, \tau_{1}\right]$ вместо $\left[t_{0}, t_{1}\right]$. Тогда для всех $\omega$, для которых $F\left(\omega^{\prime}\right) \geqslant 0$ при $t \in\left[\tau_{0}, \tau_{1}\right]$, выполнено неравенство $I(\omega) \leqslant I(\gamma)$. Действительно, предположим, что на отрезке $\left[\tau_{0}, \tau_{1}\right]$ существует такая кривая $\widetilde{\omega}$, совпадающая в его концах с $\gamma$, что $I(\widetilde{\omega})>I(\gamma)$. Определим непрерывную кривую $\omega$, совпадающую с $\widetilde{\omega}$ на отрезке $\left[\tau_{0}, \tau_{1}\right]$ и с $\gamma$ на множестве $\left[t_{0}, t_{1}\right] \backslash\left[\tau_{0}, \tau_{1}\right]$. Тогда в силу определения функционалов $I$ и $J$ получаем $J(\omega)-J(\gamma)=I(\widetilde{\omega})-I(\gamma)>0$, что противоречит доказанному выше.

Наконец, рассмотрим произвольную кривую $\omega$ на отрезке $\left[t_{0}, t_{1}\right]$, совпадающую в его концах с геодезической $\gamma$. Разобьем $\left[t_{0}, t_{1}\right]$ на части, где функция $F\left(\omega^{\prime}\right)$ знакопостоянна. Если $\left[\tau_{0}, \tau_{1}\right] \subset\left[t_{0}, t_{1}\right]-$ отрезок, внутри которого $F\left(\omega^{\prime}\right)<0$, то интеграл от $\sqrt{F\left(\omega^{\prime}\right)}$ по $\left[\tau_{0}, \tau_{1}\right]$ дает нулевой вклад в вещественную часть (39) и положительный - в мнимую. Отсюда сразу следует, что $J_{\mathrm{I}}(\omega) \geqslant J_{\mathrm{I}}(\gamma)=0$. Если же $\left[\tau_{0}, \tau_{1}\right]$ - отрезок, на котором $F\left(\omega^{\prime}\right) \geqslant 0$, то в силу доказанного выше $I(\omega) \leqslant I(\gamma)$. Суммируя интегралы по всем таким отрезкам, мы и получим требуемое неравенство $J_{\mathrm{R}}(\omega) \leqslant J_{\mathrm{R}}(\gamma)$.

Случай $\gamma \subset \mathscr{H}_{-}$рассматривается совершенно аналогично, в нем лишь $J_{\mathrm{R}}$ и

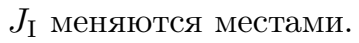

\section{§ 4. Примеры}

4.1. Пусть $S$ - гладкая поверхность, вложенная в пространство с декартовыми координатами $(x, y, z)$ и псевдоевклидовой метрикой $d s^{2}=d x^{2}+d y^{2}-d z^{2}$. В окрестности регулярной точки поверхность $S$ можно задать в виде графика гладкой функции либо от $(x, y)$, либо от $(x, z)$, либо от $(y, z)$.

Рассмотрим первый случай, обозначив $t=y$ и задав поверхность $S$ в виде $z=f(t, x)$. Тогда псевдоримановой метрике на $S$, порождаемой псевдоевклидовой метрикой объемлющего пространства, соответствует квадратичная форма (1), где

$$
a=1-f_{x}^{2}, \quad b=-f_{t}^{\prime} f_{x}^{\prime}, \quad c=1-f_{t}^{2}, \quad \Delta=f_{t}^{2}+f_{x}^{2}-1 .
$$


Из (41) видно, что в параболической точке $q$ выполнено равенство $f_{t}^{2}+f_{x}^{2}=1$, следовательно, коэффициенты $a$ и $c$ неотрицательны и геодезические, входящие в $q$ с неизотропными направлениями, являются времениподобными. Кроме того, $a+c=1$, откуда видно, что по крайней мере один из коэффициентов $a$ и $c$ отличен от нуля. Предположим, что $a \neq 0$, это равносильно условию $f_{t}^{\prime} \neq 0$.

Подставляя (41) в формулы (9), получаем

$$
\begin{gathered}
\mu_{3}=2 f_{t}^{\prime} f_{x x}^{\prime \prime}\left(1-f_{x}^{2}\right), \quad \mu_{2}=2\left(2 f_{t}^{\prime} f_{t x}^{\prime \prime}-f_{x}^{\prime} f_{x x}^{\prime \prime}\right), \\
\mu_{1}=2\left(f_{t}^{\prime} f_{t t}^{\prime \prime}-2 f_{x}^{\prime} f_{t x}^{\prime \prime}\right), \quad \mu_{0}=-2 f_{x}^{\prime} f_{t t}^{\prime \prime} .
\end{gathered}
$$

Для упрощения вычислений мы можем воспользоваться ортогональным преобразованием плоскости $(t, x)$, на которой ограничение метрики евклидово. Легко видеть, что, сделав поворот аффинной плоскости $(t, x)$ вокруг точки $q$ на подходящий угол, мы, сохраняя метрику на $S$, можем сделать $f_{x}^{\prime}(q)=0$. Тогда из (8) и (42) получаем

$$
M(q, p)=2 f_{t}^{\prime}(q) p\left[f_{x x}^{\prime \prime}(q) p^{2}+2 f_{t x}^{\prime \prime}(q) p+f_{t t}^{\prime \prime}(q)\right] .
$$

Из условия 3) типичности параболической точки $q$ следует, что изотропное направление $p_{0}=0$ не касается кривой $\mathscr{D}$, определенной уравнением $f_{t}^{2}+f_{x}^{2}=1$. Так как $f_{x}^{\prime}(q)=0$ и $f_{t}^{\prime}(q) \neq 0$, то это дает условие $f_{t t}^{\prime}(q) \neq 0$. Из формулы $(43)$ следует, что тогда $p=0$ является простым корнем многочлена $M(q, p)$. Следовательно, выполнено условие теоремы 3 и имеется семейство геодезических $\gamma_{\alpha}$, $\alpha \in \mathfrak{A}$, касающихся изотропного направления $p_{0}=0$. Из (43) также ясен геометрический смысл условий $\widetilde{D}<0$ и $\widetilde{D}>0$, определяющих случаи, когда многочлен $M(q, p)$ имеет один и три корня. Дискриминант квадратного трехчлена $f_{x x}^{\prime \prime}(q) p^{2}+2 f_{t x}^{\prime \prime}(q) p+f_{t t}^{\prime \prime}(q)$ с точностью до постоянного отрицательного сомножителя совпадает с гауссовой кривизной поверхности $S$ в точке $q$, поэтому условие $\widetilde{D}<0$ (соответственно $\widetilde{D}>0$ ) означает, что $S$ - поверхность положительной (соответственно отрицательной) кривизны. ${ }^{6}$ Если $\widetilde{D}>0$, то многочлен $M(q, p)$ имеет корень $p=0$, которому соответствует изотропное направление, и корни $\widetilde{p}_{1}$ и $\widetilde{p}_{2}$, которым соответствуют времениподобные направления, совпадающие с асимптотическими направлениями поверхности $S$ в точке $q{ }^{7}$

Второй и третий случаи совершенно симметричны. Обозначим $t=z$ и рассмотрим поверхность $S$, заданную графиком $y=f(t, x)$. Тогда псевдоримановой метрике на $S$ соответствует квадратичная форма (1), где

$$
a=f_{x}^{2}+1, \quad b=f_{t}^{\prime} f_{x}^{\prime}, \quad c=f_{t}^{2}-1, \quad \Delta=1-f_{t}^{2}+f_{x}^{2} .
$$

В параболической точке $q$ выполнено равенство $f_{t}^{2}-f_{x}^{2}=1$, коэффициенты $a$ и $c$ неотрицательны, причем $a \geqslant 1$. Геодезические, входящие в $q$ с неизотропными направлениями, времениподобны. Подставляя (44) в формулы (9), получаем

$$
\begin{gathered}
\mu_{3}=-2 f_{t}^{\prime} f_{x x}^{\prime \prime}, \quad \mu_{2}=-2\left(f_{x}^{\prime} f_{x x}^{\prime \prime}+2 f_{t}^{\prime} f_{t x}^{\prime \prime}\right), \quad \mu_{1}=-2\left(f_{t}^{\prime} f_{t t}^{\prime \prime}+2 f_{x}^{\prime} f_{t x}^{\prime \prime}\right), \\
\mu_{0}=-2 f_{x}^{\prime} f_{t t}^{\prime \prime} .
\end{gathered}
$$

\footnotetext{
63десь кривизна поверхности подразумевается в стандартной евклидовой метрике $d s^{2}=$ $d x^{2}+d y^{2}+d z^{2}$ объемлющего пространства.

${ }^{7}$ Однако, записав уравнение асимпототических линий в виде, аналогичном (6), легко видеть, что сами геодезические $\widetilde{\gamma}_{1}$ и $\widetilde{\gamma}_{2}$ с асимптотическими линиями поверхности $S$ не совпадают.
} 
Воспользуемся лоренцевым преобразованием плоскости $(t, x)$, на которой ограничение метрики псевдоевклидово. Сделав гиперболический поворот аффинной плоскости $(t, x)$ вокруг точки $q$, мы, сохраняя метрику на $S$, можем сделать $f_{x}^{\prime}(q)=0$. Тогда из $(8)$ и $(45)$ мы получаем многочлен $M(q, p)$, отличающийся от (43) лишь знаком. Все дальнейшие рассуждения повторяют сказанное в предыдущем случае и дают те же самые результаты.

4.2. Рассмотрим случай, когда все коэффициенты $a, b, c$ квадратичной формы (1) не зависят от одной из переменных $x$ и $t$. Тогда уравнение (3) и поле $(14)$ имеют интеграл импульса $L_{p}^{\prime}(t, p)$ в первом случае и интеграл энергии $H(x, p)=p L_{p}^{\prime}-L$ во втором. Покажем, что инвариантные слоения $L_{p}^{\prime}=$ const или $H=$ const вблизи особых точек $(q, \widetilde{p})$ и $\left(q, p_{0}\right)$ из теорем 2 и 3 имеют различный геометрический смысл: в окрестности $\left(q, p_{0}\right)$ они трансверсальны и дают в пересечении интегральные кривые поля, а в окрестности $(q, \widetilde{p})$ они совпадают.

Например, рассмотрим первый случай: коэффициенты не зависят от $x$. Покажем, что в окрестности точки $\left(q, p_{0}\right)$ из теоремы 3 интегральные кривые поля (14) получаются пересечением инвариантных слоений $L_{p}^{\prime}=$ const и $U=$ const. Интегральные поверхности $L_{p}^{\prime}=$ const имеют вид цилиндров с основаниями - множествами $F_{p}^{\prime} F^{-1 / 2}=$ const на плоскости $(t, p)$ - и образующими, параллельными оси $x$. Уравнения этих цилиндров можно записать в виде

$$
F+k F_{p}^{2}=0, \quad k \in \mathbb{R} \cup\{\infty\},
$$

где случай $k=\infty$ обозначает уравнение $F_{p}^{\prime}=0$. В точке $\left(q, p_{0}\right)$ обе функции $F$ и $F_{p}^{\prime}$ обращаются в нуль, поэтому все цилиндры $(46)$ проходят через точку $\left(q, p_{0}\right)$ и, следовательно, содержат прямую, проходящую через эту точку параллельно оси $x$. Так как $L$ не зависит от $x$, то $F_{x}^{\prime}=0$. В точке $\left(q, p_{0}\right)$ производная $F_{p}^{\prime}$ не обращается в нуль и, как следует из $(13), F_{t}^{\prime} \neq 0$; таким образом,

$$
\left(F+k F_{p}^{2}\right)_{x}^{\prime}=\left(F+k F_{p}^{2}\right)_{p}^{\prime}=0, \quad\left(F+k F_{p}^{2}\right)_{t}^{\prime} \neq 0 .
$$

Следовательно, в точке $\left(q, p_{0}\right)$ все цилиндры $(46)$ касаются осей $x$ и $p$. Второе инвариантное слоение $U=$ const касается поля контактных плоскостей $p d t-d x=0$, поэтому цилиндры (46) трансверсально пересекаются с проходящим через точку $\left(q, p_{0}\right)$ слоем $U=$ const (далее будем обозначать его $U=0$ ) и высекают на нем интегральные кривые поля (14) с вертикальным касательным направлением. Если интегральная кривая не вертикальна, т.е. для соответствующего значения $k$ функция $F+k F_{p}^{2}$ от переменных $(t, p)$ не обращается тождественно (по $p$ ) в нуль при подстановке $t=0$, то ее проекция на плоскость $(t, x)$ является геодезической. Дифференцируя $F+k F_{p}^{2}$ по $p$, получаем $F_{p}^{\prime}\left(1+2 k F_{p p}^{\prime \prime}\right)=2(a p+b)(1+4 k a)$. Второе дифференцирование дает $2 a(1+4 k a)$. Таким образом, при всех конечных $k \neq-\frac{1}{4} a^{-1}(q)$ сечения слоя $U=0$ цилиндрами (46) суть траектории $\gamma_{\alpha}^{\prime}$ при всех конечных $\alpha \in \mathfrak{A}$. Исключенное значение $k=-\frac{1}{4} a^{-1}(q)$ отвечает исключенному из множества $\mathfrak{A}$ значению $\alpha_{*}$ и вертикальной кривой $\gamma_{\alpha_{*}}^{\prime}$ (ось $p$ ). Так как $F_{p p}^{\prime \prime}=2 a \neq 0$, то поверхность уравнения $F_{p}^{\prime}=0$, т.е. цилиндр (46) при $k=\infty$, в точке $\left(q, p_{0}\right)$ трансверсальна слою $U=0$ и высекает на нем интегральную кривую поля (14) с касательным направлением $\vec{e}_{2 \lambda}$ из $(26)$; это и есть траектория $\gamma_{\infty}^{\prime}$. 
В точке $(q, \widetilde{p})$ из теоремы 2 функция $F$ не обращается в нуль и $\Delta=M=0$. Из условия 3) в определении типичной параболической точки следует, что $\Delta_{t}^{\prime} \neq 0$. Из формулы (8) в случае, когда $F$ не зависит от $x$, следует, что $2 F F_{t p}^{\prime \prime}-F_{t}^{\prime} F_{p}^{\prime}=$ $2 M$. С учетом этого соотношения из формулы (5) получаем выражения

$$
L_{p p}^{\prime \prime}=-\Delta F^{-3 / 2}, \quad L_{t p}^{\prime \prime}=\frac{1}{2} M F^{-3 / 2} .
$$

Так как в точке $(q, \widetilde{p})$ имеем $\Delta=M=0$, то в ней обе производные (47) обращаются в нуль. Далее, дифференцируя выражения (47) по $p$, в точке $(q, \widetilde{p})$ получаем

$$
L_{p p p}^{\prime \prime \prime}=0, \quad L_{t p p}^{\prime \prime \prime}=\frac{1}{2} M_{p}^{\prime} F^{-3 / 2}=-\Delta_{t}^{\prime} F^{-3 / 2} \neq 0 .
$$

Второе равенство основано на формуле (18). Действительно, подставляя в (18) значение $p=\widetilde{p}$, с учетом того, что $M=\Delta_{x}^{\prime}=0$ и $\widetilde{p} \neq p_{0}$, получаем $2 \Delta_{t}^{\prime}+M_{p}^{\prime}=0$. Таким образом, линейная часть функции $L_{p}^{\prime}(t, p)$ в точке $t=0, p=\widetilde{p}$ равна нулю, а квадратичная часть эквивалентна форме $\xi \eta$. Следовательно, заменой переменных $(t, p)$ интеграл импульса $L_{p}^{\prime}$ приводится к форме $\xi \eta$, т.е. он эквивалентен интегралу $U=\xi \eta$ нормальной формы $(22)$ и задает то же самое инвариантное слоение $U=$ const. $^{8}$

Во втором случае, когда все коэффициенты $a, b, c$ квадратичной формы (1) не зависят от переменной $t$, система (3) и поле (14) имеют интеграл энергии $H(x, p)$, и все рассуждения совершенно аналогичны. Можно также формально свести этот случай к первому, поменяв переменные $t$ и $x$ местами.

\section{Список литературы}

[1] А. Д. Сахаров, "Космологические переходы с изменением сигнатуры метрики", ЖЭТФ, 87:2(8) (1984), 375-383.

[2] Б. Л. Альтшулер, А. О. Барвинский, "Квантовая космология и физика переходов с изменением сигнатуры пространства-времени”, УФН, 166:5 (1996), 459-492.

[3] T. Dereli, R. W. Tucker, "Signature dynamics in general relativity", Classical Quantum Gravity, 10:2 (1993), 365-373.

[4] G. Ellis, A. Sumeruk, D. Coule, C. Hellaby, "Change of signature in classical relativity", Classical Quantum Gravity, 9:6 (1992), 1535-1554.

[5] S. A. Hayward, "Signature change in general relativity", Classical Quantum Gravity, 9:8 (1992), 1851-1862.

[6] M. Kossowski, "Pseudo-Riemannian metric singularities and the extendability of parallel transport", Proc. Amer. Math. Soc., 99:1 (1987), 147-154.

[7] M. Kossowski, M. Kriele, "Signature type change and absolute time in general relativity", Classical Quantum Gravity, 10:6 (1993), 1157-1164.

[8] M. Kossowski, M. Kriele, "Transverse, type changing, pseudo Riemannian metrics and the extendability of geodesics", Proc. Roy. Soc. London Ser. A, 444:1921 (1994), 297-306.

[9] M. Kossowski, M. Kriele, "The Einstein equation for signature type changing spacetimes", Proc. Roy. Soc. London Ser. A, 446:1926 (1994), 115-126.

\footnotetext{
${ }^{8}$ Сами функции $L_{p}^{\prime}$ и $U$ могут и не совпадать, так как замена, приводящая интеграл $L_{p}^{\prime}$ к форме $\xi \eta$, не обязательно приводит и поле (14) к нормальной форме (22). Однако геометрический смысл имеет совпадение не самих первых интегралов (которые вообе заданы не однозначно), а определяемых ими инвариантных слоений.
} 
[10] А. А. Давыдов, М. А. Комаров, "Бифуркации локальной управляемости в семействах бидинамических систем на плоскости", Дифференииальные уравнения и динамические системы, Тр. МИАН, 261, Наука, М., 2008, 87-96; англ. пер.: A. A. Davydov, M. A. Komarov, "Local controllability bifurcations in families of bidynamical systems on the plane", Proc. Steklov Inst. Math., 261:1 (2008), 84-93.

[11] А. О. Ремизов, "Особенности коразмерности 2 в трехмерных аффинно-управляемых системах со скалярным управлением”, Матем. сб., 199:4 (2008), 143-158; англ. пер.: А. O. Remizov, "Codimension-two singularities in 3D affine control systems with a scalar control", Sb. Math., 199:4 (2008), 613-627.

[12] В. И. Арнольд, Дополнительные главы теории обыкновенных дифференииальных уравнений, Наука, M., 1978; фр. пер.: V. Arnol'd, Chapitres supplémentaires de la théorie des équations différentielles ordinaires, Mir, Moscow, 1980.

[13] В. И. Арнольд, Геометрические методы в теории обыкновенных дифференциальных уравнений, Изд-во Удмуртского ун-та, Ижевск, 2000.

[14] В. И. Арнольд, Ю. С. Ильяшенко, "Обыкновенные дифференциальные уравнения", Динамические системъ - 1, Итоги науки и техн. Сер. Соврем. пробл. мат. Фундам. направления, 1, ВИНИТИ, М., 1985, 7-140; англ. пер.: V. I. Arnol'd, Yu. S. Il'yashenko, "Ordinary differential equations", Dynamical systems I, Encyclopaedia Math. Sci., 1, Springer-Verlag, Berlin, 1988, 7-140.

[15] А.А. Давыдов, "Нормальная форма дифференциального уравнения, не разрешенного относительно производной, в окрестности его особой точки", Функи. анализ и его прил., 19:2 (1985), 1-10; англ. пер.: А. A. Davydov, "Normal form of a differential equation, not solvable for the derivative, in a neighborhood of a singular point", Functional Anal. Appl., 19:2 (1985), 81-89.

[16] А.О. Ремизов, "Многомерная конструкция Пуанкаре и особенности поднятых полей для неявных дифференциальных уравнений”, СМФН, 19 (2006), 131-170; англ. пер.: А. O. Remizov, "Multidimensional Poincaré construction and singularities of lifted fields for implicit differential equations", J. Math. Sci. (N. Y.), 151:6 (2008), 3561-3602.

[17] R. Roussarie, "Modèles locaux de champs et de formes", Astérisque, 30 (1975), 1-181.

[18] А. Д. Брюно, Локальный метод нелинейного анализа дифференииальных уравнений, Наука, М., 1979; англ. пер.: А. D. Bruno, Local methods in nonlinear differential equations, Springer Ser. Soviet Math., Springer-Verlag, Berlin, 1989.

[19] В. В. Немыцкий, В.В. Степанов, Качественная теория дифференииальных уравнений, ГИТТЛ, М.-Л., 1949; англ. пер.: V. V. Nemytskii, V. V. Stepanov, Qualitative theory of differential equations, Princeton Univ. Press, Princeton, NJ, 1960.

[20] S. M. Voronin, "The Darboux-Whitney theorem and related questions", Nonlinear Stokes phenomena, Adv. Soviet Math., 14, Amer. Math. Soc., Providence, RI, 1993, 139-233.

[21] С. М. Воронин, “Аналитическая классификация ростков голоморфных отображений с неизолированными неподвижными точками и постоянными мультипликаторами и ее приложения", Вестник ЧелГУ. Сер. 3. Матем., мех., 2(5):3 (1999), 12-30.

[22] А. С. Мищенко, А. Т. Фоменко, Курс дифференциальной геометрии и топологии, 2-е изд., Факториал, М., 2000; англ. пер. 1-го изд.: А. Mishchenko, A. Fomenko, A course of differential geometry and topology, Mir, Moscow, 1988.

[23] М.И. Зеликин, Оптимальное управление и вариачионное исчисление, УРСС, М., 2004. 